\title{
无配体参与钯催化烯烃与芳基三烷氧基硅烷的交叉偶联反应
}

\author{
唐伯孝* 方小牛国仁云 周小春 \\ (井冈山大学化学化工学院 应用化学研究所 江西省配位化学重点实验室 吉安 343009)
}

\begin{abstract}
摘要 考察了钯催化下一系列烯烃化合物与不同芳基三烷氧基硅烷的反应, 发展了一种新型无配体参与的 Heck 类型 反应方法. 研究表明: 以 $\mathrm{Pd}(\mathrm{OAc})_{2}$ 为催化剂, 在 $\mathrm{K}_{2} \mathrm{CO}_{3}, n-\mathrm{Bu}_{4} \mathrm{NF} \cdot 3 \mathrm{H}_{2} \mathrm{O}$ 和 $\mathrm{AgOAc}$ 存在下, 该 Heck 类型反应能够得到 中等及优良的偶联产率, 并且表现出较高的区域选择性和立体选择性. 根据实验结果以及相关文献, 对该 Heck 类型偶 联反应的可能机理进行了探讨.
\end{abstract}

关键词 无配体; 钯催化; Heck 类型反应; 芳基三烷氧基硅烷

\section{Ligand-Free Pd-Catalyzed Cross-Coupling Reaction of Olefins and Trialkoxyarylsilanes}

\author{
Tang, Boxiao* Fang, Xiaoniu Kuang, Renyun Zhou, Xiaochun \\ (School of Chemistry and Chemical Engineering, Institute of Applied Chemistry, and Key Laboratory of Coordination \\ Chemistry of Jiangxi Province, Jinggangshan University, Ji'an 343009)
}

\begin{abstract}
A ligand-free Pd-catalyzed method was developed for Heck-type reaction of a wide range of olefins with various trialkoxyarylsilanes. In the presence of $\mathrm{Pd}(\mathrm{OAc})_{2}, \mathrm{~K}_{2} \mathrm{CO}_{3}, \mathrm{TBAF} \bullet 3 \mathrm{H}_{2} \mathrm{O}$ and $\mathrm{AgOAc}$, the cross-coupling products can be obtained in moderate to good yields, and the Heck-type reaction displayed high regio- and stereo-selectivities. The mechanism of Heck-type reaction was discussed according to our experimental results and the reported mechanism.

Keywords ligand-free; palladium-catalyst; Heck-type reaction; trialkoxyarylsilanes
\end{abstract}

过渡金属催化的反应已经成为构建碳一碳、碳一杂 原子等化学键的有效手段, 在现代有机合成中扮演着不 可或缺的关键角色 ${ }^{[1]}$. 在这些研究方法中, Heck 反应因 为良好的化学选择性与催化效率, 逐渐发展成为一种应 用日益广泛的有机合成方法 ${ }^{[2]}$, 尤其在具有生理活性天 然产物、药物合成以及新型材料制备等领域有着重要的 实际运用价值. 传统的 Heck 反应一般是指烯烃与卤代 物发生的偶联反应 ${ }^{[3]}$. 随着人们对 Heck 反应的逐步深 入了解，其他底物替代卤化物的 Heck 反应也被人们所 认识. Hiyama 等 ${ }^{[4]}$ 研究人员利用硅醇类化合物替代卤化 物找到了一些新型高效 Heck 反应催化体系. 近年来, 钯催化烯烃与芳基三烷氧基硅烷的交叉偶联反应也引 起人们的研究兴趣. 2009 年, Loh 等 ${ }^{[5]}$ 以乙酰胺作为导向 基团, 通过 $\mathrm{C}-\mathrm{H}$ 键的功能化, 成功实现了 $\mathrm{C}-\mathrm{C}$ 键的构 建(图 1 中式 1). Mino 和 Cheng 等 ${ }^{[6]}$ 利用氮配体, 在烯烃
底物选择上有所突破，实现了一般烯烃与芳基三烷氧基 硅烷的交叉偶联反应(图 1 中式 2). 上述研究方法，虽 然在选择性和产品收率上具有显著优势，但偶联反应对 导向基团、配体的依赖性却制约有机硅化物 Heck 类型 反应的广泛运用.

本文以 $\mathrm{Pd}(\mathrm{OAc})_{2}$ 为催化剂, 在无配体加入的条件 下，研究了一般烯烃与芳基三烷氧基硅化物的交叉偶联 反应，并获得了理想的实验结果(图 1 中式 3).

\section{1 结果与讨论}

\section{1 反应条件的优化}

我们以苯基三甲氧基硅烷(1a)和丙烯酸叔丁酯(2a) 的反应为模板，在 $5 \% \mathrm{Pd}(\mathrm{OAc})_{2}$ 的条件下，对各种反应 条件进行篮选(如表 1 所示): 首先, 我们考察了不同溶 剂对反应的影响，在一系列有机溶剂中，1,2-二氯乙烷

\footnotetext{
*E-mail: 520tangboxiao@163.com

Received October 7, 2012; revised November 15, 2012; published online November 20, 2012.

Project supported by the Science and Technology Project of Jiangxi Provincial Department of Education (No. GJJ12471) and the Doctoral Program of Jinggangshan University (No. JZ10042).

江西省教育厅科技(No. GJJ12471)和井冈山大学博士启动基金(No. JZ10042)资助项目.
} 


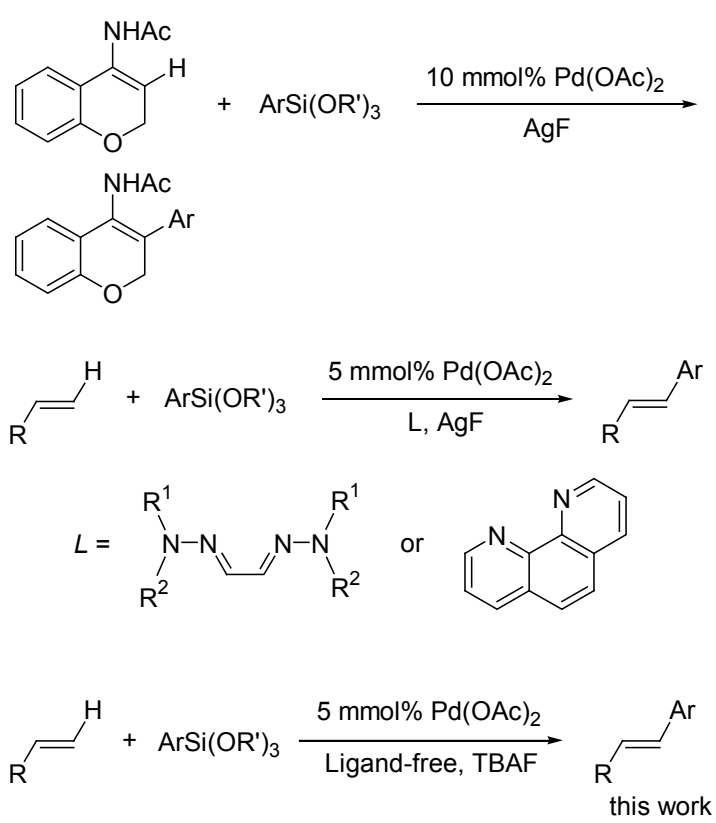

图 1 钯催化芳基三烷氧基硅烷 Heck 类型反应

Figure 1 Pd-catalyzed Heck-type reaction of trialkoxyarylsilanes

表 1 苯基三甲氧基硅烷与丙烯酸叔丁酯的 Heck 反应 ${ }^{a}$

Table 1 Heck-type reaction of trimethoxy(phenyl)silane with tert-butylacrylate

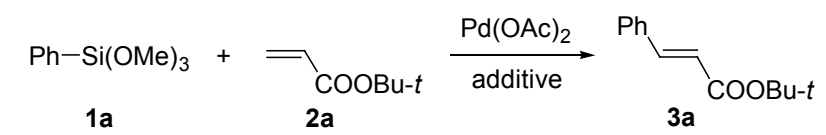

\begin{tabular}{|c|c|c|c|c|}
\hline Entry & Oxidant & Solvent & Base & Yield $^{b} \%$ \\
\hline 1 & $\mathrm{AgOAc}$ & Acetonitrile & $\mathrm{K}_{2} \mathrm{CO}_{3}$ & 58 \\
\hline 2 & $\mathrm{AgOAc}$ & Toluene & $\mathrm{K}_{2} \mathrm{CO}_{3}$ & 62 \\
\hline 3 & $\mathrm{AgOAc}$ & DMF & $\mathrm{K}_{2} \mathrm{CO}_{3}$ & 44 \\
\hline 4 & $\mathrm{AgOAc}$ & THF & $\mathrm{K}_{2} \mathrm{CO}_{3}$ & 50 \\
\hline 5 & $\mathrm{AgOAc}$ & Methanol & $\mathrm{K}_{2} \mathrm{CO}_{3}$ & $<10$ \\
\hline 6 & $\mathrm{AgOAc}$ & 1,4-Dioxane & $\mathrm{K}_{2} \mathrm{CO}_{3}$ & 58 \\
\hline 7 & $\mathrm{AgOAc}$ & DCE & $\mathrm{K}_{2} \mathrm{CO}_{3}$ & 84 \\
\hline $8^{c}$ & $\mathrm{AgOAc}$ & DCE & $\mathrm{K}_{2} \mathrm{CO}_{3}$ & Trace \\
\hline $9^{d}$ & $\mathrm{AgOAc}$ & DCE & $\mathrm{K}_{2} \mathrm{CO}_{3}$ & 11 \\
\hline $10^{e}$ & $\mathrm{AgOAc}$ & DCE & $\mathrm{K}_{2} \mathrm{CO}_{3}$ & 65 \\
\hline $11^{f}$ & $\mathrm{AgOAc}$ & DCE & $\mathrm{K}_{2} \mathrm{CO}_{3}$ & 70 \\
\hline 12 & $\mathrm{AgOAc}$ & DCE & KOAc & 50 \\
\hline 13 & $\mathrm{AgOAc}$ & DCE & $\mathrm{NaOH}$ & 54 \\
\hline 14 & $\mathrm{AgOAc}$ & DCE & $\mathrm{NaOAc}$ & 72 \\
\hline 15 & $\mathrm{AgOAc}$ & DCE & - & Trace \\
\hline 16 & $\mathrm{AgNO}_{3}$ & DCE & $\mathrm{K}_{2} \mathrm{CO}_{3}$ & 61 \\
\hline 17 & $\mathrm{Ag}_{2} \mathrm{O}$ & DCE & $\mathrm{K}_{2} \mathrm{CO}_{3}$ & 63 \\
\hline 18 & $\mathrm{Ag}_{2} \mathrm{CO}_{3}$ & DCE & $\mathrm{K}_{2} \mathrm{CO}_{3}$ & 51 \\
\hline 19 & - & DCE & $\mathrm{K}_{2} \mathrm{CO}_{3}$ & 0 \\
\hline $20^{c}$ & $\mathrm{AgF}$ & DCE & $\mathrm{K}_{2} \mathrm{CO}_{3}$ & 8 \\
\hline
\end{tabular}

${ }^{a}$ Reaction condition: 1a $(0.3 \mathrm{mmol}), \mathbf{2 a}(0.2 \mathrm{mmol}), \mathrm{Pd}(\mathrm{OAc})_{2}(5 \mathrm{~mol} \%)$, $\mathrm{TBAF} \cdot 3 \mathrm{H}_{2} \mathrm{O}$ (TBAF $=$ tetra- $n$-butylammoniu fluoride) (2 equiv.), $[\mathrm{Ag}](2$ equiv.), base (2 equiv.), solvent ( $1 \mathrm{~mL}$ ), $8 \mathrm{~h}, 80{ }^{\circ} \mathrm{C}, \mathrm{N}_{2} \cdot{ }^{b}$ Isolated yield. ${ }^{c}$ No TBAF $\bullet 3 \mathrm{H}_{2} \mathrm{O} .{ }^{d}$ Room temperature. ${ }^{e} 60{ }^{\circ} \mathrm{C} .{ }^{f}$ Air.
(DCE) 表现出最好的反应活性(表 1, Entries 1 7); 当反 应体系没有氟盐或者碱时，仅得到痕量的目标产物(表 1, Entries 8，15); 同时, 考察了不同温度对偶联反应的 影响，在室温和 $60{ }^{\circ} \mathrm{C}$ 的情况下分别得到 $11 \%$ 和 $65 \%$ 的 收率(表 1, Entries 9,10); 当用空气代替氮气时，催化效 果略有下降(表 1, Entry 11); 接下来考察了不同碱对偶 联反应的影响, 实验结果表明 $\mathrm{K}_{2} \mathrm{CO}_{3}$ 的产率最高(表 1 , Entries 5,12,13，14); 最后, 各种银盐的笁选实验结果 表明 $\mathrm{AgOAc}$ 的催化效果最佳, 没有任何银盐时偶联反 应不能发生(表 1, Entries 7, 16 19), 当用 $\mathrm{AgF}$ 代替 $\mathrm{AgOAc}$ 和 TBAF 时, 偶联产率仅得到 8\% (表 1, Entry 20).

\section{2 烯烃与芳基三烷氧基硅烷的交叉偶联反应}

通过模板反应的研究，我们找到了偶联反应最优的 条件: 芳基三烷氧基硅烷 1a $(0.3 \mathrm{mmol})$, 烯烃 2a $(0.2$ $\mathrm{mmol}), \mathrm{Pd}(\mathrm{OAc})_{2}(5 \mathrm{~mol} \%), \mathrm{TBAF} \bullet 3 \mathrm{H}_{2} \mathrm{O}(0.4 \mathrm{mmol})$, AgOAc $(0.4 \mathrm{mmol}), \mathrm{K}_{2} \mathrm{CO}_{3}(0.4 \mathrm{mmol}), \mathrm{DCE}(1 \mathrm{~mL}), 8 \mathrm{~h}$, $80{ }^{\circ} \mathrm{C}, \mathrm{N}_{2}$. 为了探究该催化体系的适用范围, 我们选择 了一系列烯烃与不同芳基三烷氧基硅化合物进行偶联 反应，实验结果见表 2 . 从表 2 可以看出: 不同类型的烯 烃都可以得到中等以上的收率，含吸电子基团烯烃的偶 联反应效果要好些(表 2, Entries 1 5); 当芳基和硅烷上 有不同取代基时，偶联反应的活性也有些差异(表 2 , Entries 6 13).

\section{3 反应机理的探讨}

根据所得实验结果以及相关文献报道 ${ }^{[7,4 a]}$, 我们对 芳基三烷氧基硅烷参与的 Heck 类型反应可能发生的机 理进行探讨(图 2): 在实验过程中反应体系不加 TBAF 时, 反应几乎不能发生, 这说明偶联反应首先应该在氟 盐的活化下形成中间体 $\mathbf{1}$; 然后，中间体 $\mathbf{1}$ 与 $\mathrm{Pd}(\mathrm{OAc})_{2}$

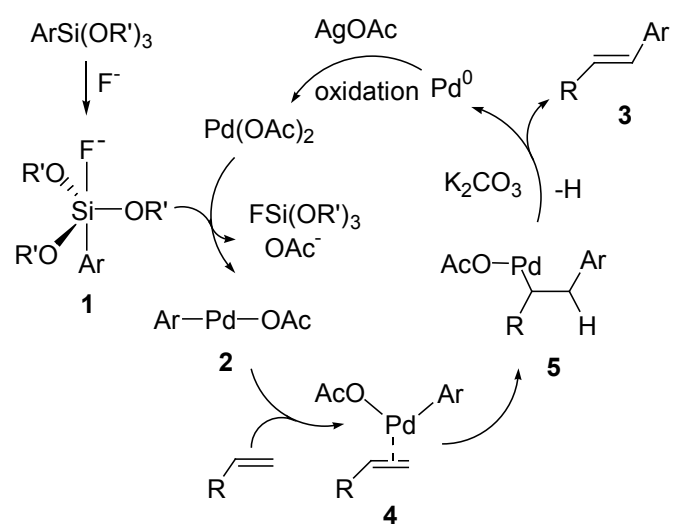

图 2 可能的反应机理

Figure 2 A possible mechanism 
表 2 无配体钯催化芳基三烷氧基硅烷与烯烃的 Heck 反应 ${ }^{a}$

Table 2 Ligand-free Pd-catalyzed Heck reaction of trialkoxyarylsilanes with olefins

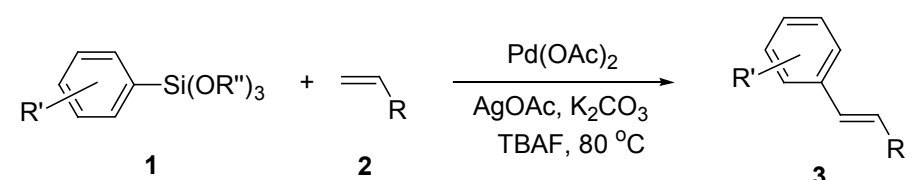

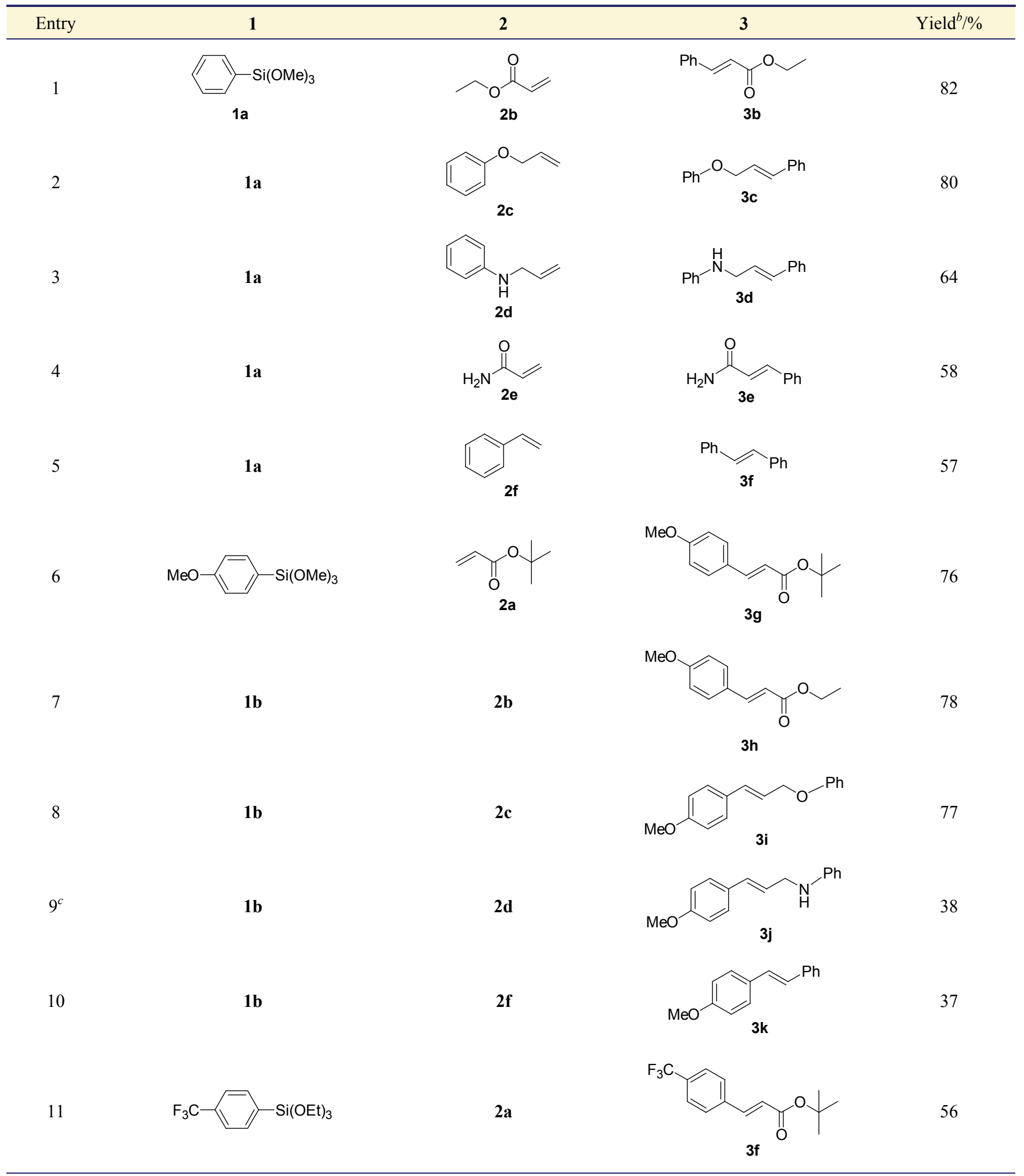




\begin{tabular}{|c|c|c|c|c|}
\hline Entry & 1 & 2 & 3 & Yield $^{b} / \%$ \\
\hline 12 & $1 \mathrm{c}$ & $2 \mathrm{~b}$ & $3 m$ & 58 \\
\hline 13 & $1 \mathrm{c}$ & $2 c$ & & 63 \\
\hline
\end{tabular}

${ }^{a}$ Reaction condition: 1a $(0.3 \mathrm{mmol}), \mathbf{2 a}(0.2 \mathrm{mmol}), \mathrm{Pd}(\mathrm{OAc})_{2}(5 \mathrm{~mol} \%), \mathrm{TBAF} \bullet 3 \mathrm{H}_{2} \mathrm{O}$ (2 equiv.), $\mathrm{AgOAc}\left(2\right.$ equiv.), $\mathrm{K}_{2} \mathrm{CO}_{3}(2$ equiv. $), \mathrm{DCE}(1 \mathrm{~mL}), 8 \mathrm{~h}, 80{ }^{\circ} \mathrm{C}, \mathrm{N}_{2}$

${ }^{b}$ Isolated yield. ${ }^{c} 12 \mathrm{~h}$.

作用得到钯化合物 2; 接着, 金属钯化合物 2 与烯烃的 $\pi$ 键配位形成中间体 4; 随后, 芳基和钯分别加成在烯烃 的 $\alpha$-碳和 $\beta$-碳上(良好的区域选择性与立体选择性证明 了这一点), 得到中间体 $\mathbf{5}$; 在碱的作用下, 中间体 $\mathbf{5}$ 还 原消除得到目标产物 $\mathbf{3}$ 和 $\mathrm{Pd}^{0}$ (反应体系中没有碱时只能 得到痕量的目标产物); 最后, 零价钯在氧化剂的作用 下又形成二价钯(没有银盐反应不能发生), 至此, 钯催 化剂完成了整个催化循环.

\section{2 结论}

本文发展了一种新型无配体钯催化芳基三烷氧基 硅烷参与的 Heck 类型反应方法. 一系列研究结果表明: 在 $\mathrm{Pd}(\mathrm{OAc})_{2}(5 \mathrm{~mol} \%), \mathrm{TBAF} \bullet 3 \mathrm{H}_{2} \mathrm{O}(0.4 \mathrm{mmol}), \mathrm{AgOAc}$ $(0.4 \mathrm{mmol}), \mathrm{K}_{2} \mathrm{CO}_{3}(0.4 \mathrm{mmol}), \mathrm{DCE}(1 \mathrm{~mL})$ 的反应条件 下, 各种烯烃与不同的芳基三烷氧基硅烷能够得到中等 及优良的偶联产率, 而且反应有着较好的区域选择性和 立体选择性. 根据实验结果探讨了这类 Heck 反应的可 能机理, 这对有机硅化合物 Heck 类型反应深入研究是 有益的发展.

\section{3 实验部分}

\section{1 仪器与试剂}

所用的药品和试剂均为市售的分析纯或化学纯, 未 进一步处理. 柱层析使用 $300 \sim 400$ 目硅胶. 核磁共振 仪: Bruker AVANCE III, 以 TMS 为内标, 気代氯仿为溶 剂. 熔点: 样品装在毛细管中在石蜡油中缓慢加热测的, 温度未校正.

\section{2 实验方法}

在干燥的 Schlenk 管中加入烷氧基硅烷 $(0.3 \mathrm{mmol})$, 烯烃 $(0.2 \mathrm{mmol})$, 醋酸钯 $(0.015 \mathrm{mmol})$, 氟盐 $(0.4 \mathrm{mmol})$, 银盐 $(0.4 \mathrm{mmol})$, 碱 $(0.4 \mathrm{mmol})$, 溶剂 $(1 \mathrm{~mL}), \mathrm{N}_{2}$ 气氛中, 在 $80{ }^{\circ} \mathrm{C}$ 油浴中加热反应 $8 \mathrm{~h}$. 反应结束后, 降至室温, 加入乙醚, 将反应物转入圆底烧瓶, 浓缩, 上硅胶柱, 以石油醚/乙酸乙酯为洗脱剂分离提纯得到目标产物.

\section{3 产物结构表征}

(E)-3-苯基丙烯酸叔丁酯(3a) ${ }^{[8 a]}$ : 淡黄色油状物; ${ }^{1} \mathrm{H}$ NMR (500 MHz, $\left.\mathrm{CDCl}_{3}\right) \delta: 7.53(\mathrm{~d}, J=16.0 \mathrm{~Hz}, 1 \mathrm{H}), 7.44$ (m, 2H), $7.31 \sim 7.29(\mathrm{~m}, 3 \mathrm{H}), 6.31(\mathrm{~d}, J=16.0 \mathrm{~Hz}, 1 \mathrm{H})$, $1.46(\mathrm{~s}, 9 \mathrm{H}) ;{ }^{13} \mathrm{C}$ NMR $\left(125 \mathrm{MHz}, \mathrm{CDCl}_{3}\right) \delta: 166.3,143.5$, 134.7, 129.9, 128.8, 127.9, 120.2, 80.5, 28.2.

$(E)$-3-苯基丙烯酸乙酯 $(\mathbf{3 b})^{[8]}$ : 无色油状物; ${ }^{1} \mathrm{H}$ NMR $\left(500 \mathrm{MHz}, \mathrm{CDCl}_{3}\right) \delta: 7.63(\mathrm{~d}, J=16.0 \mathrm{~Hz}, 1 \mathrm{H})$, $7.46 \sim 7.45$ (m, $2 \mathrm{H}), 7.32 \sim 7.31(\mathrm{~m}, 3 \mathrm{H}), 6.38$ (d, $J=16.0$ $\mathrm{Hz}, 1 \mathrm{H}), 4.22 \sim 4.17(\mathrm{~m}, 2 \mathrm{H}), 1.38(\mathrm{t}, J=7.0 \mathrm{~Hz}, 3 \mathrm{H}) ;{ }^{13} \mathrm{C}$ NMR $\left(125 \mathrm{MHz}, \mathrm{CDCl}_{3}\right) \delta: 167.0,144.6,134.5,130.2$, $128.9,128.1,118.3,60.5,14.3$.

(E)-1-苯基-3-苯氧基丙烯(3c): 白色固体, m.p. 64 $65{ }^{\circ} \mathrm{C}$ (lit. ${ }^{[8 b]} 62 \sim 63{ }^{\circ} \mathrm{C}$ ); ${ }^{1} \mathrm{H}$ NMR $\left(500 \mathrm{MHz}, \mathrm{CDCl}_{3}\right) \delta$ : 7.34 (d, $J=7.5 \mathrm{~Hz}, 2 \mathrm{H}), 7.24 \sim 7.22(\mathrm{~m}, 3 \mathrm{H}), 7.20 \sim 7.17$ $(\mathrm{m}, 2 \mathrm{H}), 6.89(\mathrm{~d}, J=7.5 \mathrm{~Hz}, 3 \mathrm{H}), 6.67(\mathrm{~d}, J=16.0 \mathrm{~Hz}$, $1 \mathrm{H}), 6.37 \sim 6.31(\mathrm{~m}, 1 \mathrm{H}), 4.62(\mathrm{~d}, J=6.0 \mathrm{~Hz}, 2 \mathrm{H}) ;{ }^{13} \mathrm{C}$ NMR $\left(125 \mathrm{MHz}, \mathrm{CDCl}_{3}\right) \delta: 158.6,136.4,132.9,129.5$, 128.6, 127.9, 126.5, 124.5, 120.9, 114.7, 68.5.

(E)- $N$-肉桂基苯胺 $(\mathbf{3 d})^{[8 c]}$ : 淡黄色油状物; ${ }^{1} \mathrm{H}$ NMR $\left(500 \mathrm{MHz}, \mathrm{CDCl}_{3}\right) \delta: 7.29$ (t, $\left.J=8.0 \mathrm{~Hz}, 2 \mathrm{H}\right), 7.24(\mathrm{t}, J=$ $7.5 \mathrm{~Hz}, 2 \mathrm{H}), 7.18 \sim 7.15(\mathrm{~m}, 2 \mathrm{H}), 7.14 \sim 7.10(\mathrm{~m}, 2 \mathrm{H})$, $6.72 \sim 6.67(\mathrm{~m}, 3 \mathrm{H}), 6.56(\mathrm{~d}, J=16.0 \mathrm{~Hz}, 1 \mathrm{H}), 6.29 \sim 6.23$ (m, 1H), $3.87(\mathrm{~d}, J=5.5 \mathrm{~Hz}, 2 \mathrm{H}) ;{ }^{13} \mathrm{C}$ NMR $(125 \mathrm{MHz}$, $\left.\mathrm{CDCl}_{3}\right) \delta: 146.9,136.8,132.3,129.3,128.6,127.6,126.4$, 126.2, 118.7, 114.1, 47.0.

$(E)$-肉桂酰胺 $(3 \mathrm{e}))^{[8 \mathrm{dd}]}$ : 淡黄色油状物; ${ }^{1} \mathrm{H}$ NMR (500 $\left.\mathrm{MHz}, \mathrm{CDCl}_{3}\right) \delta: 7.61(\mathrm{~d}, J=15.5 \mathrm{~Hz}, 1 \mathrm{H}), 7.44 \sim 7.43(\mathrm{~m}$, $2 \mathrm{H}), 7.30 \sim 7.29(\mathrm{~m}, 3 \mathrm{H}), 6.48(\mathrm{~d}, J=16.0 \mathrm{~Hz}, 1 \mathrm{H}), 6.35$ (br s, $2 \mathrm{H}) ;{ }^{13} \mathrm{C}$ NMR $\left(125 \mathrm{MHz}, \mathrm{CDCl}_{3}\right) \delta: 168.8,143.8$, 134.2, 130.4, 128.9, 128.2, 118.6.

(E)-1,2-二苯基乙烯(3f): 白色固体, m.p. 122 123 ${ }^{\circ} \mathrm{C}$ (lit. ${ }^{[8]}$ m.p. $\left.123 \sim 124{ }^{\circ} \mathrm{C}\right)$; ${ }^{1} \mathrm{H}$ NMR $(500 \mathrm{MHz}$, $\left.\mathrm{CDCl}_{3}\right) \delta: 7.44(\mathrm{~d}, J=8.0 \mathrm{~Hz}, 4 \mathrm{H}), 7.29(\mathrm{t}, J=7.5 \mathrm{~Hz}, 4 \mathrm{H})$, 
$7.19 \sim 7.15(\mathrm{~m}, 2 \mathrm{H}), 7.03(\mathrm{~s}, 2 \mathrm{H}) ;{ }^{13} \mathrm{C}$ NMR (125 MHz, $\left.\mathrm{CDCl}_{3}\right) \delta: 137.4,128.8,128.7,127.6,126.5$.

$(E)-3$-对甲氧基苯基丙烯酸叔丁酯 $(\mathbf{3 g})^{[8 \mathrm{e}]}$ : 淡黄色 油状物; ${ }^{1} \mathrm{H}$ NMR (500 MHz, $\left.\mathrm{CDCl}_{3}\right) \delta: 7.48(\mathrm{~d}, J=15.5$ $\mathrm{Hz}, 1 \mathrm{H}), 7.39$ (d, $J=8.5 \mathrm{~Hz}, 2 \mathrm{H}), 6.82$ (d, $J=8.5 \mathrm{~Hz}, 2 \mathrm{H})$, $6.18(\mathrm{~d}, J=16.0 \mathrm{~Hz}, 1 \mathrm{H}), 3.75(\mathrm{~s}, 3 \mathrm{H}), 1.45(\mathrm{~s}, 9 \mathrm{H}) ;{ }^{13} \mathrm{C}$ NMR $\left(125 \mathrm{MHz}, \mathrm{CDCl}_{3}\right) \delta: 166.7,161.1,143.2,129.5$, 127.3, 117.6, 114.2, 80.2, 55.3, 28.2.

(E)-3-对甲氧基苯基丙烯酸乙酯 $(3 h){ }^{[8 f]}$ : 淡黄色油 状物; ${ }^{1} \mathrm{H} \mathrm{NMR}\left(500 \mathrm{MHz}, \mathrm{CDCl}_{3}\right) \delta: 7.65(\mathrm{~d}, J=16.0 \mathrm{~Hz}$, $1 \mathrm{H}), 7.48$ (d, $J=7.5 \mathrm{~Hz}, 2 \mathrm{H}), 6.90$ (d, $J=7.5 \mathrm{~Hz}, 2 \mathrm{H}), 6.32$ $(\mathrm{d}, J=15.5 \mathrm{~Hz}, 1 \mathrm{H}), 4.27 \sim 4.23(\mathrm{~m}, 2 \mathrm{H}), 1.34$ (t, $J=7.0$ $\mathrm{Hz}, 3 \mathrm{H}) ;{ }^{13} \mathrm{C} \mathrm{NMR}\left(125 \mathrm{MHz}, \mathrm{CDCl}_{3}\right) \delta: 167.3,161.3$, $144.2,129.6,127.1,115.7,114.3,60.3,55.3,14.3$.

(E)-1-对甲氧基苯基-3-苯氧基丙烯(3i): 白色固体, m.p. $102 \sim 103{ }^{\circ} \mathrm{C}$ (lit. ${ }^{[8 b]}$ m.p. 103 $\sim 104{ }^{\circ} \mathrm{C}$ ); ${ }^{1} \mathrm{H}$ NMR $\left(500 \mathrm{MHz}, \mathrm{CDCl}_{3}\right) \delta: 7.27(\mathrm{~d}, J=8.5 \mathrm{~Hz}, 2 \mathrm{H}), 7.22(\mathrm{~d}, J=$ $8.0 \mathrm{~Hz}, 2 \mathrm{H}), 6.89$ (d, $J=7.0 \mathrm{~Hz}, 3 \mathrm{H}), 6.78(\mathrm{~d}, J=8.5 \mathrm{~Hz}$, $2 \mathrm{H}), 6.60(\mathrm{~d}, J=16.0 \mathrm{~Hz}, 1 \mathrm{H}), 6.22(\mathrm{~d}, J=16.0 \mathrm{~Hz}, 1 \mathrm{H})$, $4.58(\mathrm{~d}, J=5.5 \mathrm{~Hz}, 2 \mathrm{H}), 3.71(\mathrm{~s}, 3 \mathrm{H}) ;{ }^{13} \mathrm{C} \mathrm{NMR}(125 \mathrm{MHz}$, $\left.\mathrm{CDCl}_{3}\right) \delta: 159.5,158.7,132.8,129.5,129.2,127.9,122.2$, 120.9, 114.8, 114.0, 68.6, 55.3.

$(E)-N$-对甲氧基肉桂基苯胺 $(\mathbf{3 j})^{[\mathrm{gg}]}$ : 淡黄色油状物; ${ }^{1} \mathrm{H}$ NMR $\left(500 \mathrm{MHz}, \mathrm{CDCl}_{3}\right) \delta: 7.22(\mathrm{~d}, J=8.0 \mathrm{~Hz}, 2 \mathrm{H})$, $7.13(\mathrm{~d}, J=7.5 \mathrm{~Hz}, 2 \mathrm{H}), 6.77$ (d, $J=8.0 \mathrm{~Hz}, 2 \mathrm{H}), 6.68$ (t, $J=8.0 \mathrm{~Hz}, 1 \mathrm{H}), 6.63(\mathrm{~d}, J=8.0 \mathrm{~Hz}, 2 \mathrm{H}), 6.49$ (d, $J=15.5$ $\mathrm{Hz}, 1 \mathrm{H}), 6.13 \sim 6.08(\mathrm{~m}, 1 \mathrm{H}), 3.82(\mathrm{~d}, J=5.5 \mathrm{~Hz}, 2 \mathrm{H})$, $3.71(\mathrm{~s}, 4 \mathrm{H}) ;{ }^{13} \mathrm{C} \mathrm{NMR}\left(125 \mathrm{MHz}, \mathrm{CDCl}_{3}\right) \delta: 159.2,147.6$, $131.4,129.3,127.8,127.6,124.3,118.1,114.2,114.0$, $55.3,46.7$.

(E)-1-苯基-2-对甲氧基苯基乙烯(3k): 淡黄色固体, m.p. $134 \sim 135{ }^{\circ} \mathrm{C}$ (lit. ${ }^{[8 f]}$ m.p. $136 \sim 138{ }^{\circ} \mathrm{C}$ ); ${ }^{1} \mathrm{H}$ NMR $\left(500 \mathrm{MHz}, \mathrm{CDCl}_{3}\right) \delta: 7.40(\mathrm{~d}, J=7.5 \mathrm{~Hz}, 2 \mathrm{H}), 7.36(\mathrm{~d}, J=$ $7.5 \mathrm{~Hz}, 2 \mathrm{H}), 7.26(\mathrm{t}, J=7.5 \mathrm{~Hz}, 2 \mathrm{H}), 7.15(\mathrm{t}, J=6.0 \mathrm{~Hz}$, $1 \mathrm{H}), 6.98$ (d, $J=16.5 \mathrm{~Hz}, 1 \mathrm{H}), 6.89$ (d, $J=16.0 \mathrm{~Hz}, 1 \mathrm{H})$, $6.80(\mathrm{~d}, J=7.5 \mathrm{~Hz}, 2 \mathrm{H}), 3.71(\mathrm{~s}, 3 \mathrm{H}) ;{ }^{13} \mathrm{C} \mathrm{NMR}(125 \mathrm{MHz}$, $\left.\mathrm{CDCl}_{3}\right) \delta: 159.4,137.7,130.2,128.7,128.3,127.8,127.3$, 126.7, 126.3, 114.2, 55.4.

(E)-3-对三氟甲基苯基丙烯酸叔丁酯 $(3 \mathrm{l})^{[8 \mathrm{e}]}$ : 淡黄色 油状物; ${ }^{1} \mathrm{H}$ NMR (500 MHz, $\left.\mathrm{CDCl}_{3}\right) \delta: 7.56 \sim 7.50(\mathrm{~m}$, $5 \mathrm{H}), 6.38$ (d, $J=16.0 \mathrm{~Hz}, 1 \mathrm{H}), 1.47$ (s, 9H); ${ }^{13} \mathrm{C}$ NMR $(125$ $\left.\mathrm{MHz}, \mathrm{CDCl}_{3}\right) \delta: 165.7,141.7,138.1,131.6,128.7,125.8$, 125.0, 122.8, 81.0, 28.1.

(E)-3-对三氟甲基苯基丙烯酸乙酯 $(\mathbf{3 m})^{[8 \mathrm{f}]}$ : 淡黄色 油状物; ${ }^{1} \mathrm{H}$ NMR (500 MHz, $\mathrm{CDCl}_{3}$ ) $\delta: 7.63$ (d, $J=16.0$
$\mathrm{Hz}, 1 \mathrm{H}), 7.58 \sim 7.54(\mathrm{~m}, 4 \mathrm{H}), 6.45(\mathrm{~d}, J=16.0 \mathrm{~Hz}, 1 \mathrm{H})$, $4.23 \sim 4.19(\mathrm{~m}, 2 \mathrm{H}), 1.29(\mathrm{t}, J=7.5 \mathrm{~Hz}, 3 \mathrm{H}) ;{ }^{13} \mathrm{C} \mathrm{NMR}$ $\left(125 \mathrm{MHz}, \mathrm{CDCl}_{3}\right) \delta: 166.4,142.7,137.8,131.8,128.7$, 125.7, 122.7, 120.8, 60.8, 14.3.

(E)-1-对三氟甲基苯基-3-苯氧基丙烯 $(3 n)$ : 淡黄色 固体, m.p. $65 \sim 67{ }^{\circ} \mathrm{C}$ (lit. ${ }^{[8 b]}$ m.p. $60 \sim 63{ }^{\circ} \mathrm{C}$ ); ${ }^{1} \mathrm{H}$ NMR $\left(500 \mathrm{MHz}, \mathrm{CDCl}_{3}\right) \delta: 7.51(\mathrm{~d}, J=8.5 \mathrm{~Hz}, 2 \mathrm{H}), 7.43(\mathrm{~d}, J=$ $8.0 \mathrm{~Hz}, 2 \mathrm{H}), 7.25(\mathrm{t}, J=8.0 \mathrm{~Hz}, 2 \mathrm{H}), 6.90(\mathrm{~d}, J=8.5 \mathrm{~Hz}$, $3 \mathrm{H}), 6.71(\mathrm{~d}, J=16.0 \mathrm{~Hz}, 1 \mathrm{H}), 6.46 \sim 6.41(\mathrm{~m}, 1 \mathrm{H}), 4.65$ $(\mathrm{d}, J=5.0 \mathrm{~Hz}, 2 \mathrm{H}) ;{ }^{13} \mathrm{C} \mathrm{NMR}\left(125 \mathrm{MHz}, \mathrm{CDCl}_{3}\right) \delta: 158.5$, $140.0,131.2$, 129.8, 129.0, 127.4, 126.7, 125.6, 123.1, $121.1,114.7,68.1$.

\section{References}

[1] (a) de Meijere, A.; Diederich, F. Metal-Catalyzed Cross-Coupling Reactions, Wiley-VCH, Weinheim, Germany, 2004.

(b) Beller, M.; Bolm, C. Transition Metals for Organic Synthesis, 2nd ed., Wiley-VCH, Weinheim, Germany, 2004.

(c) Ritleng, V.; Sirlin, C.; Pfeffer, M. Chem. Rev. 2002, 102, 1731.

(d) Xu, Q.; Zhao, C.; Zhou, Y.; Yin, S.; Han, L. Chin. J. Org. Chem. 2012, 32, 1761 (in Chinese).

(徐清, 赵长秋, 周永波, 尹双风, 韩立彪, 有机化学, 2012, 32, 1761.)

(e) Magano, J.; Dunet, J. R. Chem. Rev. 2011, 111, 2177.

(f) Xie, Y.; Song, R.; Xiang, J.; Li, J. Chin. J. Org. Chem. 2012, 32, 1555 (in Chinese).

(谢叶香, 宋仁杰, 向建南, 李金恒, 有机化学, 2012, 32, 1555.)

[2] (a) Oestreich, M. The Mizoroki-Heck Reaction, Wiley, Chichester, UK, 2009.

(b) Miyaura, N. Cross-Coupling Reaction, Springer, Berlin, 2002.

(c) Beletskaya, I. P.; Cheprakov, A. V. Chem. Rev. 2000, 100, 3009.

(d) Nicolaou, K. C.; Sorensen, E. J. Classics in Total Synthesis, VCH, New York, 1996, Chapter 31.

(e) Link, J. T.; Overman, L. E. In Metal-Catalyzed Cross-Coupling Reactions, Eds.: Diederich, F.; Stang, P. J., Wiley-VCH, New York, 1998, Chapter 6.

[3] (a) Heck, R. F.; Nolley, J. P. J. Org. Chem. 1972, 37, 2320.

(c) Ikeda, Y.; Nakamura, T.; Yorimitsu, H.; Oshima, K. J. Am. Chem. Soc. 2002, 124, 6514.

(d) Li, J.-H.; Wang, D.-P.; Xie, Y.-X. Synthesis 2005, 2193.

(e) Ren, G.; Cui, X.; Yang, E.; Yang, F.; Wu, Y. Tetrahedron 2010, 66, 4022 .

(f) Wu, X.; Pei, W. Chin. J. Chem. 2009, 27, 963.

(g) Zhou, R.; Yu, X.; Wang, W.; Fu, H.; Li, R.; Chen, H.; Li, X. Chin. J. Org. Chem. 2011, 31, 1490 (in Chinese).

(周蓉, 余晓军, 汪伟, 付海燕, 李瑞祥, 陈华, 李贤均, 有机化 学, 2011, 31, 1490.)

[4] (a) Hirabayashi, K.; Nishihara, Y.; Mori, A.; Hiyama, T. Tetrahedron Lett. 1998, 39, 7893.

(b) Hirabayashi, K.; Ando, J.; Kawashima, J.; Nishihara, Y.; Mori, A.; Hiyama, T. Bull. Chem. Soc. Jpn. 2000, 73, 1409.

[5] Zhou, H.; Xu, Y.-H.; Chung, W.-J.; Loh, T.-P. Angew. Chem., Int. Ed. 2009, 48, 5355 .

[6] (a) Mino, T.; Shibuya, M.; Suzuki, S.; Hirai, K.; Sakamoto, M.; Fujita, T. Tetrahedron 2012, 68, 429.

(b) Ye, Z.; Chen, F.; Luo, F.; Wang, W.; Lin, B.; Jia, X.; Cheng, J. 
Synlett 2009, 2198.

[7] (a) Amatore, C.; Jutand, A. Acc. Chem. Res. 2000, 33, 314

(b) Nakao, Y.; Hiyama, T. Chem. Soc. Rev. 2011, 40, 4893.

[8] (a) Masllorens, J.; Moreno-Manas, M.; Pla-Quintana, A.; Roglans, A. Org. Lett. 2003, 5, 1559.

(b) Mino, T.; Shindo, H.; Kaneda, T.; Koizumi, T.; Kasashima, Y.; Sakamoto, M.; Fujita, T. Tetrahedron Lett. 2009, 50, 5358. (c) Usui, I.; Schmidt, S.; Keller, M.; Breit, B. Org. Lett. 2008, 10, 1207.

(d) Yoo, K. S.; Yoon, C. H.; Jung, K. W. J. Am. Chem. Soc. 2006, 128,16384 .

(e) Zhu, M.-K.; Zhao, J.-F.; Loh, T.-P. Org. Lett. 2011, 13, 6308

(f) Zhou, L.; Wang, L. Synthesis 2006, 2653.

(g) Nishina, N.; Yamamoto, Y. Tetrahedron 2009, 65, 1799.

(Li, L.; Fan, Y.) 